\title{
Optimisation of component performance via structuring
}

\author{
Verena Psyk ${ }^{1, \mathrm{a}}$, Petr Kurka ${ }^{1}$, Simon Kimme ${ }^{1}$, Markus Werner ${ }^{1}$, Dirk Landgrebe ${ }^{1}$, Andreas Ebert ${ }^{2}$, \\ and Mathias Schwarzendahl ${ }^{2}$ \\ ${ }^{1}$ Fraunhofer Institute for Machine Tools and Forming Technology, Reichenhainer Strasse 88, \\ 09126 Chemnitz, Germany \\ ${ }^{2}$ Westfalia Presstechnik GmbH \& Co. KG, Gewerbering 26, 08451 Crimmitschau, Germany
}

\begin{abstract}
Ecological and economic reasons are forcing industry to improve efficiency and to save energy and resources by reducing product weight. In current product designs often insufficient geometric stiffness of the part prohibits exploiting the full potential of weight reduction offered by modern materials. Ideally adapting the geometry to the load profile by implementing appropriate structures often allows a wall thickness and weight reduction and improves the acoustic properties. To enable a target-oriented design, structures manufactured by working media and working energy based forming technologies were analysed.
\end{abstract}

\section{Introduction and motivation}

Due to climate change and global warming, industry is being forced to take ecological aspects into consideration when developing new products. Over the last 20 years, Europe's transport sector has been responsible for approximately 30\% of Europe's energy consumption making it a key player in climate and resource aspects [1]. Decreasing the weight of a standard passenger car by about $100 \mathrm{~kg}$ can result in fuel savings of about 300 to 800 litres over the vehicle's lifetime and to a reduction of the $\mathrm{CO}_{2}$ emission by 9 grams per kilometre [2]. Therefore, especially for the automotive and transport industry - but also for other industrial branches - lightweight design is a key issue for improving efficiency and accordingly saving energy and resources. Developments as e.g. the multi-material concept implemented in the Audi A3 [3] already succeeded in reducing vehicle weight, so that since 2000, a trend of decreasing greenhouse gas emissions can be observed in Europe (compared to 1990) [1]. Nevertheless, according to [4] almost all car manufacturers will miss the European carbon emission reduction targets for 2020, so that further cutting of vehicle weight is indispensable.

In addition to the substitution of conventional materials by typical lightweight ones such as aluminium and magnesium alloys, high-strength steels, and plastics, also the component design offers enormous weight saving potential. When dimensioning a component, the material strength is a relevant aspect, but often insufficient geometric stiffness of the part prohibits exploiting the full potential of wall

\footnotetext{
${ }^{\text {a }}$ Corresponding author: verena.psyk@iwu.fraunhofer.de
}

This is an Open Access article distributed under the terms of the Creative Commons Attribution License 4.0, which permits unrestricted use, distribution, and reproduction in any medium, provided the original work is properly cited. 


\begin{tabular}{|c|c|c|c|}
\hline \multicolumn{4}{|c|}{ Analysis of influences } \\
\hline $\begin{array}{l}\text { Semi-finished part properties } \\
\text { - Material } \\
\text { - Thickness } \\
\text { - Pre-strain, residual stress, } \\
\text { curvature } \\
\text { (e.g. due to a preceeding } \\
\text { manufacturing process) }\end{array}$ & $\begin{array}{l}\text { Process related issues } \\
\text { - Technology choice } \\
\text { - Technology dependant } \\
\text { process parameters } \\
\text { - Tool parameters }\end{array}$ & $\begin{array}{l}\text { Structure properties } \\
\text { - Size and geometry } \\
\text { of the structuring } \\
\text { elements } \\
\text { - Distribution of the } \\
\text { structuring depth } \\
\text { - Thickness distribution }\end{array}$ & $\begin{array}{l}\text { Part performance } \\
\text { - Geometric stiffness } \\
\text { - Acoustic frequency } \\
\text { response }\end{array}$ \\
\hline
\end{tabular}

Figure 1. Analysis and application oriented design of the structuring process.

thickness reduction offered by modern high-strength materials. If the component geometry is optimally adapted to the load profile by implementing appropriate structures, a reduction in wall thickness is possible. At the same time the acoustic properties can frequently be improved.

The optimum exploitation of these effects requires a sophisticated choice and design of the structuring process. To enable an efficient and requirement dependant product and production planning, the influence of different process parameters on the resulting properties of regular hexagonal comb structures with different comb sizes and on the related part performance was systematically analysed (compare Fig. 1). Working media and working energy based processes were applied for structuring a square area of $220 \times 220 \mathrm{~mm}$.

The techniques regarded in the study feature technological and economic advantages compared to conventional sheet metal forming processes (e.g. deep drawing). One of the most important technical benefits is the improved formability of many materials due to the process specific load cases and tribological systems. Significant cost savings are possible, because in the regarded processes only one tool needs to be adapted to the specific structure, while the second active part of a conventional set of tools is replaced by the working medium and in case of the working energy based electromagnetic forming (EMF) by the tool coil, which can be flexibly applied for forming different structures. Additionally, both technologies can be integrated into a conventional sheet metal forming process and used for local structuring of deep drawn parts.

\section{Working media based structuring}

In working media based forming (also called hydroforming) a pressurised fluid, a viscous medium, or an elastomer forms tubular [5] or sheet metal [6] workpieces against a solid tool. With regard to the planned integration of the structuring process into a conventional sheet forming technology, the most promising process variants are forming with an elastomer tool and hydro-mechanical deep drawing with a membrane. One of the most important advantages is that compared to active hydroforming the complexity of the equipment is relatively low. In forming with an elastomer, the punch consists of an elastomer block enclosed by a steel frame to avoid undesired bulging. In hydro-mechanical deep drawing the fluid basin is integrated in the forming press. In both cases the pressure is generated directly in the tool due to the punch movement and the sheet workpiece is formed without necessitating an external pressurisation unit. Moreover, these processes prevent direct contact of workpiece and fluid, so that sealing effort is reduced and additional cleaning and drying is avoided [7]. In the study, structuring with an elastomer tool was chosen for investigation and comparison to parts structured by EMF. 
a)
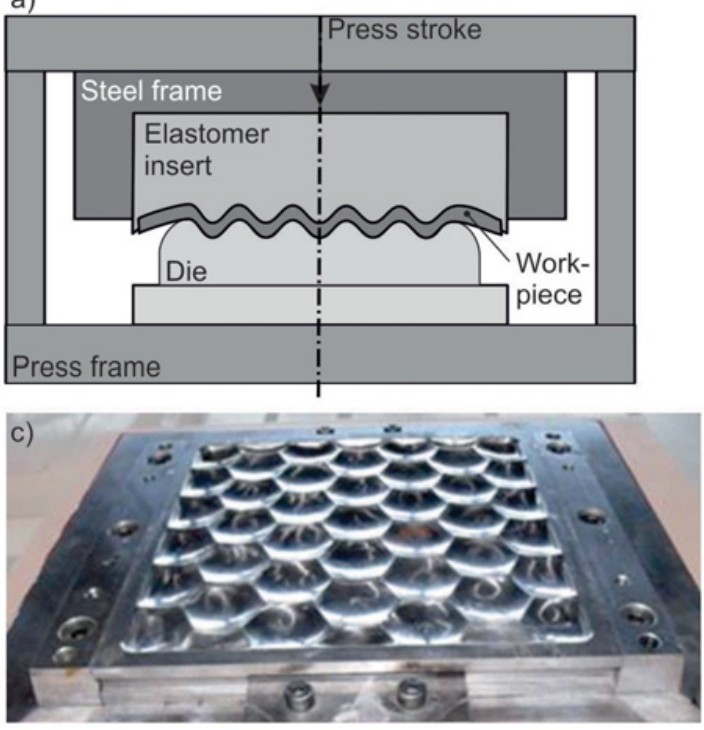

b)
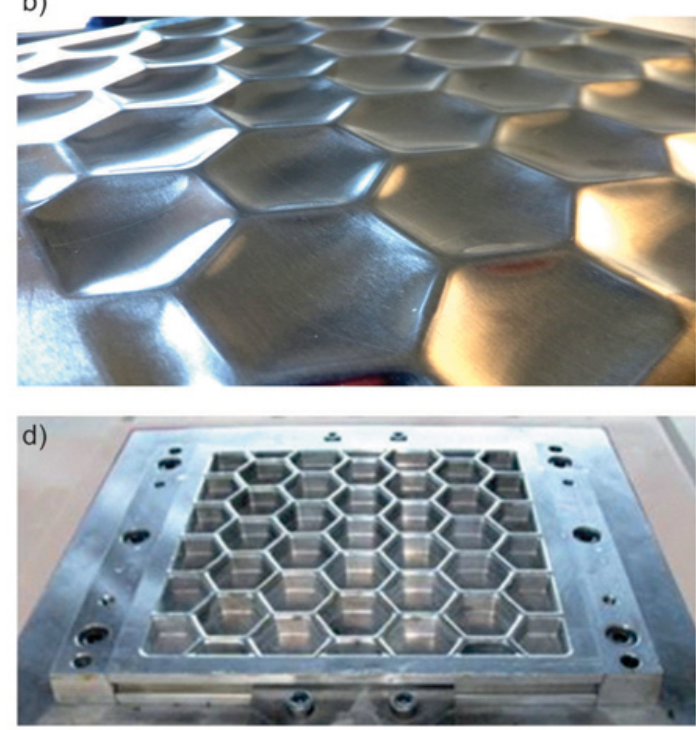

Figure 2. a) Setup of structuring with an elastomer tool; b) Example of a structured part; c) Full shape forming die; d) Forming die designed as partial structure.

\subsection{Structuring with an elastomer tool}

Structuring with an elastomer tool is a forming technology using an elastomer insert in the punch, which shapes the sheet metal workpiece (see Fig. 2). The lower die is usually designed as a conventional steel tool. The form defining surface can be realised either as a full shape solution as shown in Fig. $2 \mathrm{c}$ or as a partial structure as shown Fig. $2 d$. In the first case the complete geometry of the structure including the structure depth is defined by the die. In the second case, the die consists of $5 \mathrm{~mm}$ thick vertical division walls between the individual combs allowing free forming of the comb domes according to the chosen process parameters. This means especially that the structuring depth can by varied e.g. via the punch stroke during forming with elastomer tools. The edges of the division walls were rounded with a radius of $2.5 \mathrm{~mm}$ to avoid shearing of the workpiece, here.

In both cases the elastomer applies a pressure to the workpiece during closing of the tools which causes the sheet to align to the lower die according to the structuring geometry. In doing so, flat as well as slightly curved workpices and workpiece sections can be structured. Thereby, the application of an elastomer punch offers several advantages compared to conventional deep drawing with a set of classical full steel tools. The most important ones are that the elastomer insert applies the forming forces very uniformly, thus decreasing the stress gradient in the workpiece and consequently reducing local sheet thinning. The resulting strain distribution is more uniform compared to conventional deep drawing and the maximum strain values are lower. Consequently, the forming area features more remaining formability, which is related to higher energy absorption potential. This is of interest especially for crash relevant components from the automotive and transport industry.

Compared to the flat initial material, the stiffening structures in the formed sheet shift the originally low natural acoustic frequencies to higher values if the part is stimulated. As a result of this, the awkward low frequency noise, which is typical for thin-walled parts, such as e.g. seat components or panels, is significantly reduced. At the same time the structured material features damping properties so that less structure-borne noise is emitted. 
a)

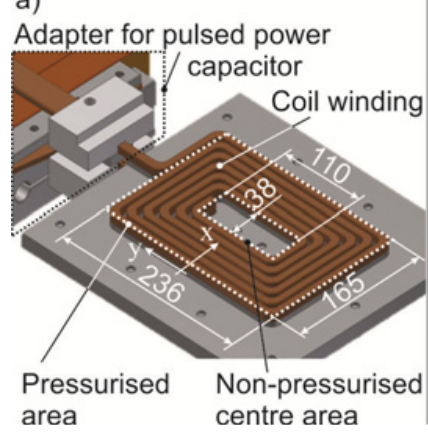

b)

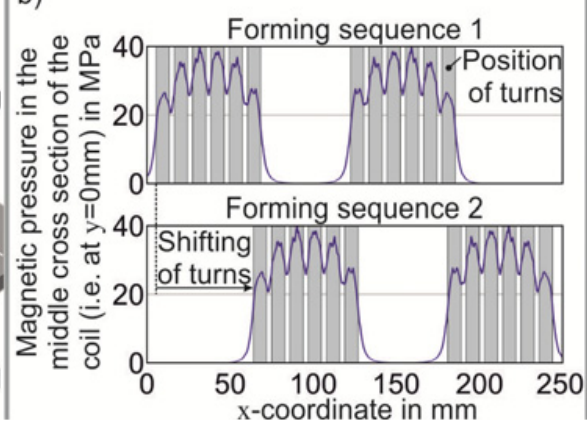

C)

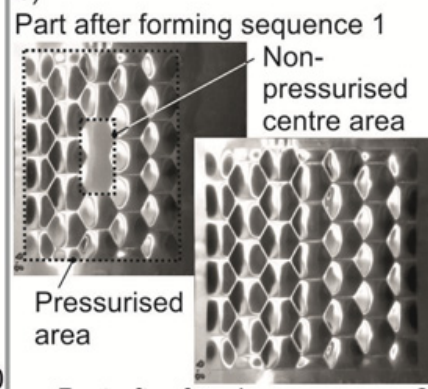

Part after forming sequence 2

Figure 3. a) Tool coil for structuring by EMF; b) Position of turns and according magnetic pressure distribution during forming sequences 1 and 2; c) Structured part after forming sequences 1 and 2.

The implementation of structures is not limited to regular geometries, but it is also possible to apply arbitrary designs including bionic ones that are inspired e.g. by cobwebs or the wings of a dragonfly. Depending on the structuring depth, especially the buckling strength can be significantly increased compared to flat sheets and component sections. Based on the material choice, the sheet thickness, and the geometry and size of the structuring elements, the bending stiffness can reach three-times the value of a flat sheet. This innovative forming technology using an elastomer punch offers numerous improvements considering the part properties, on the one hand, and can easily be implemented in classical forming tools, on the other hand.

\section{Structuring by electromagnetic forming}

\subsection{Principle and setup of structuring by EMF}

EMF is a high-speed forming process using the energy density of pulsed magnetic fields to apply Lorenz forces to workpieces made of electrically highly conductive materials as e.g. EN AW-5754, which is regarded in these investigations. The physically correct volume forces can be mathematically transferred to a so-called magnetic pressure, which is often more convenient for comparing the acting loads in different processes. Depending on the geometry and the arrangement of tool coil and workpiece, tubular parts can be compressed or expanded and forming of flat or preformed sheet metal components is possible. A comprehensive review of the technology is presented in [8].

For the investigation of structuring by EMF, a tool coil with rectangular winding geometry was designed, realised, and tested with partial structuring dies as exemplarily shown in Fig. 2d. The geometry of the coil winding is shown in Fig. 3a. In principle, the pressure distribution applied for structuring should be uniform, but in EMF only the areas of the workpiece close to the coil winding are pressurised, so that the coil considered here features an unpressurised area in the centre as it is known from spirally wound tool coils (see e.g. [8]). To overcome this deficit, the structuring was performed in two forming sequences. After the first sequence, the workpiece was moved relative to the coil, so that the zone initially positioned in the non-pressurised centre area of the coil was shifted to the pressurised area in the second forming sequence (see Fig. 3b). As shown in Fig. 3c the workpiece features undeformed regions after the first forming sequence, which are structured during the forming sequence 2 . This sequential forming approach can be used for stepwisely structuring even large flat sheets or flat sections of hollow bodies (if accessible for the coil) with various shapes of the structuring elements using one and the same coil. 

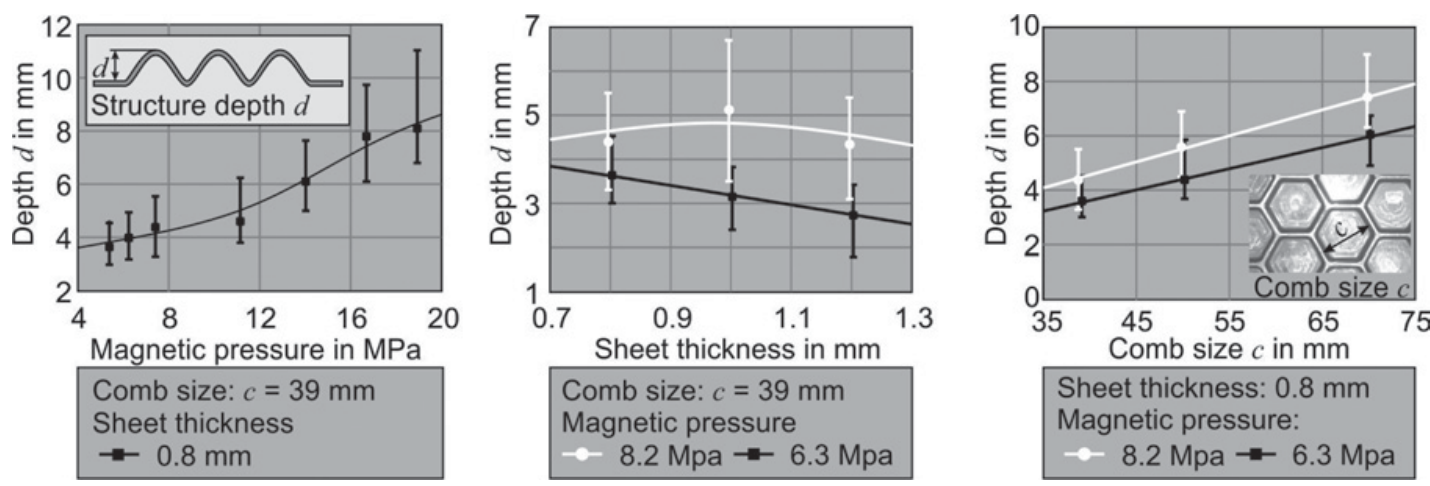

Figure 4. Influences on the structuring depth.

\subsection{Properties of structures formed by EMF}

To characterise the resulting structures, the structuring depth $d$-i.e. the depth of the comb domes (see Fig. 4) - and the wall thickness distribution was measured. In Fig. 4 the structuring depth is correlated to the maximum acting magnetic pressure, the initial sheet thickness, and the comb size.

As expected, for one and the same sheet thickness a higher pressure leads to deeper structures. The comparison of different thicknesses shows that in case of low pressure (6.3 MPA) higher depths are achieved for thinner sheets, while this trend is not so clear for higher pressures. This might be explained as follows: At the beginning, the deformation is driven by the magnetic pressure and consequently, thinner sheets are deformed more easily due to their lower inertia. After the first acceleration of the sheet, the magnetic pressure collapses quickly and the deformation is continued due to inertia effects, (see also [8]). During this process phase, the higher inertia of thicker sheets has more potential to proceed the deformation. The total structuring depth is a result of both, the pressure driven and the inertia driven deformation. Therefore, there is a pressure dependant sheet thickness leading to maximum deformation (i.e. optimum efficiency of the energy transfer). A variation of the comb size shows that larger combs feature lower resistance against forming (i.e. lower stiffness), so that deeper structures are achieved for one and the same pressure.

Compared to the parts structured with an elastomer tool, specimens structured by EMF feature a relatively high spreading of the individual comb depths even within one and the same part. This is probably owed to the specific pressure distribution and to the sequential forming approach. The qualitative wall-thickness distribution of parts structured by EMF and such structured with an elastomer punch is similar, but in case of EMF localised thinning and cracking occurs at significantly higher depths. Thus, EMF allows deeper structures compared to forming with elastomer tools, indicating an extended material formability for this high velocity forming process, an effect that is well known from previous publications as e.g. [9].

\subsection{Performance of parts structured by EMF}

To investigate the performance of the structured sheets, bending tests and measurements of the acoustic frequency response were carried out. As a reference, also unstructured sheets (i.e. structuring depth $d=0 \mathrm{~mm}$ ) were included in the measurement. The setup of the bending tests is shown in Fig. 5 . The force was applied in the centre cross section of the part at those positions where the bending punch contacts a comb edge. Force-displacement-curves were recorded during bending. These curves initially rise linearly, indicating an elastic deformation of the sheet before a further non-linear rise indicates 

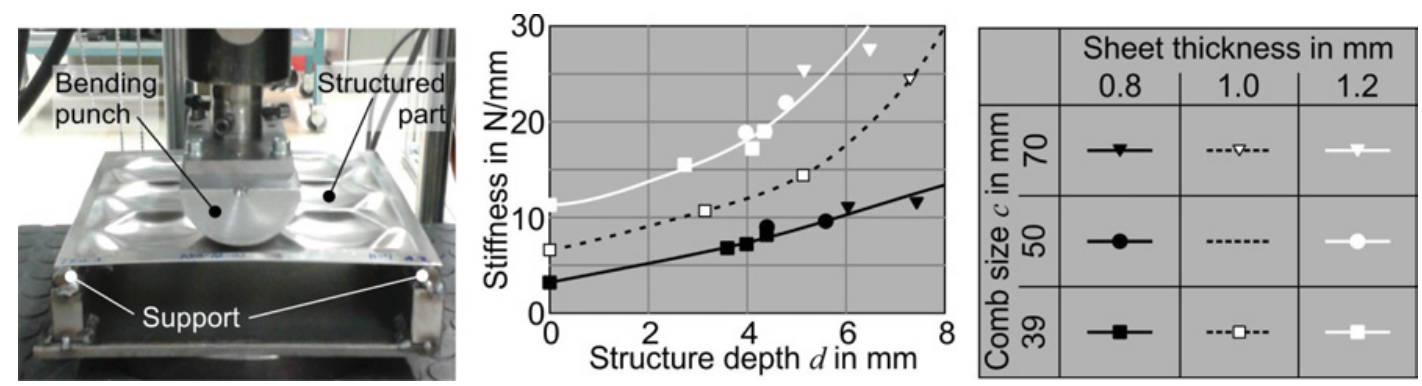

Figure 5. Geometric stiffness of specimens structured by EMF.
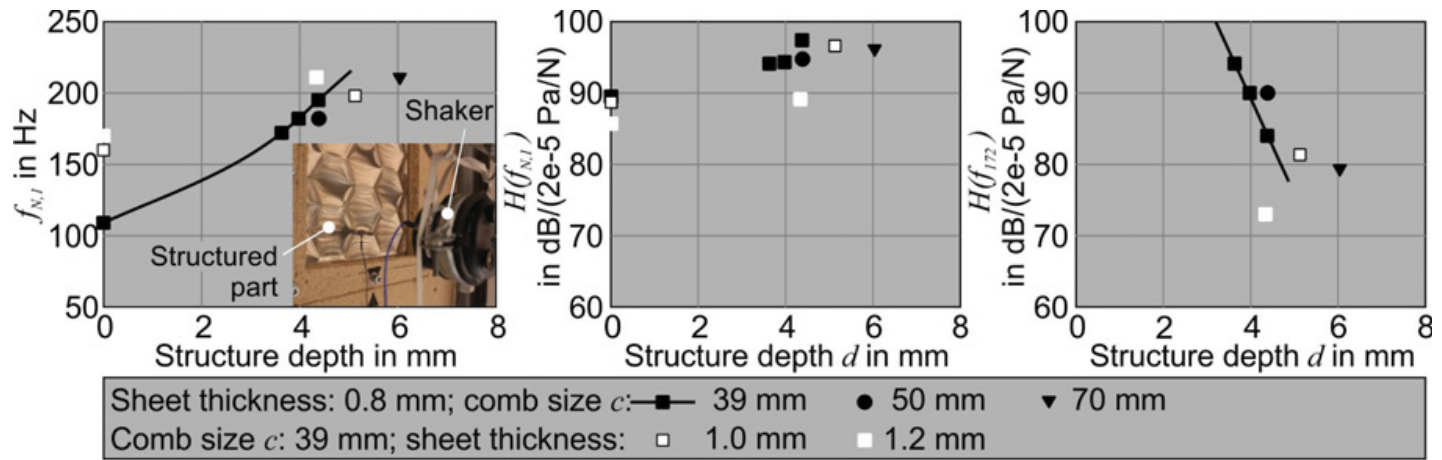

v $70 \mathrm{~mm}$

Figure 6. Acoustic properties of specimens structured by EMF.

plastic deformation. The slope of the linear section was chosen to describe the workpiece stiffness. As shown in Fig. 5, this stiffness value is higher in case of higher sheet thicknesses and rises with increasing structure depth. The influence of the comb size seems to be secondary compared to the structure depth. The comparison shows that for the regarded specimens, the wall thickness can be reduced from $1.2 \mathrm{~mm}$ to $0.8 \mathrm{~mm}$ without losing stiffness if a structure of at least $6 \mathrm{~mm}$ depth is achieved. This leads to weight savings of more than $30 \%$.

For the measurement of the acoustic part properties, the structure was excited by noise coupled in by a electro dynamic shaker. The frequency response function from exciting force to sound pressure was measured in a defined distance from the structure. Special attention was paid to the influence of the structuring on the first natural frequency $\left(f_{\mathrm{n}, 1}\right)$, the according frequency response $H\left(f_{\mathrm{n}, 1}\right)$, and the frequency response at an exemplarily chosen frequency of $172 \mathrm{~Hz} H\left(f_{172}\right)$. This frequency was selected since it is the lowest first natural frequency of the regarded structured parts. The measurement results clearly show that in general the structuring leads to a shifting of $f_{n, 1}$ to higher values, thus reducing awkward, low frequency noise in practise (see Fig. 6). Higher structure depth leads to higher $f_{\mathrm{n}, 1}$ but at the same time $H\left(f_{\mathrm{n}, 1}\right)$ increases, too. With increasing comb size deeper structures are needed to achieve the same level of $f_{\mathrm{n}, 1}$, but this does not mean that higher pressure is required during EMF (compare Fig. 4). Assuming the same structure geometry, thicker sheets cause higher values of $f_{\mathrm{n}, 1}$. The values of $H\left(f_{\mathrm{n}, 1}\right)$ are relatively similar for all regarded structures, whereas $H\left(f_{172}\right)$ shows clear dependencies. It drops severely with increasing structure depth so that significant noise reduction in low frequency range can be expected if conventional components are replaced by structured ones. Higher comb sizes again necessitate deeper structures (but not necessarily higher pressures during EMF) to achieve a similar level of $H\left(f_{172}\right)$. Higher sheet thickness also tends to result in lower $H\left(f_{172}\right)$. 


\section{Summary and outlook}

To provide fundamental knowledge for a target oriented design of structures, the influences of different parameters on the resulting structure properties and the according part performance was investigated for structuring using an elastomer punch and by EMF, respectively. The high weight saving potential (here more than $30 \%$ ) was exemplarily proved.

Future work will focus on developing a methodology using numerical simulation technologies, which allows an efficient design of local structures and the according structuring process including the selection of the most suitable forming technology, appropriate process parameters, and eligible tool properties. It will enable dimensioning application and load oriented local structures in sheet metal components leading to weight reduction and improved acoustic performance of the product. Additionally, the integration of the structuring into a conventional forming operation will be regarded.

The published research results were partly achieved within the project "Lokal versteifte Leichtbaukomponenten" (locally reinforced lightweight components), funded by the Sächsische Aufbaubank SAB with financial means of the Europäischer Fonds für Regionale Entwicklung EFRE.

\section{References}

[1] European Union (editor), EU energy in figures - Statistical pocketbook 2013, doi:10.2833/15026

[2] H. Helms, U. Lambrecht, et al. Energy savings by light-weighting. Final report for the International Aluminium Institute, Institut für Energie- und Umweltforschung GmbH. Heidelberg (2003)

[3] AUDI AG (editor), Workshop Audi A3 (2012) https : //www . audi-mediaservices . com

[4] T. Lawrence, Developing vehicles to meet carbon emissions reduction targets (2015), http://www.paconsulting.com/our-thinking/developing-vehicles-to-meet-carbonemissions-reduction-targets/

[5] M. Koç, T. Altan, J. Mat. Proc. Tech. 108, 384-393 (2001)

[6] S.H. Zhang, Z.R. Wang, Y. Xu, Z.T. Wang, L.X. Zhou, J. Mat. Proc. Tech. 151, 237-241 (2004)

[7] M. Beckmann, F. Vollertsen, J. Mat. Proc. Tech. 174, 363-370 (2006)

[8] V. Psyk, D. Risch, B.L. Kinsey, A.E. Tekkaya, M. Kleiner, J. Mat. Proc. Tech. 211, 787-829 (2011)

[9] Golovashchenko, S., J. Mater. Eng. Perform. 16 (3), 314-320 (2007) 